\title{
Impacts of Machine Learning on Work
}

\author{
Kevin Crowston \\ Syracuse University \\ School of Information Studies \\ crowston@syr.edu
}

\author{
Francesco Bolici \\ Universit degli Studi di Cassino \\ Department of Economy and Law \\ f.bolici@unicas.it
}

\begin{abstract}
The increased pervasiveness of technological advancements in automation makes it urgent to address the question of how work is changing in response. Focusing on applications of machine learning (ML) that automate information tasks, we present a simple framework for identifying the impacts of an automated system on a task. From an analysis of popular press articles about ML, we develop 3 patterns for the use of ML: decision support, blended decision making and complete automation. We further consider how automation of one task might have implications for other tasks. Our main conclusion is that designers have a range of options for systems and that automation of tasks is not the same as automation of work.
\end{abstract}

\section{Introduction}

Work has constantly evolved in response to the increased capabilities of technologies supporting human activities. However, the interlinked evolution of work design and technology has been accelerated by the quick expansion of the technological capabilities of artificial intelligence (AI), machine learning (ML) in particular. ML can support the automation of a broad range of activities, even decision-making tasks that until recently were the exclusive domain of humans. For example, ML is being applied to tasks ranging from credit-card fraud detection [1], to detecting skin cancers [2], to advising in judicial decisions [3].

Much of the rhetoric around work and AI focuses on people being replaced by automated systems. However, this view of the relationship between people and machines is too simplistic, because automatable tasks rarely stand in isolation [4]. As a result, analysts expect that "technological disruptions such as robotics and machine learning — rather than completely replacing existing occupations and job categories - are likely to substitute specific tasks previously carried out as part of these jobs" [5]. But the impact of such partial automation needs to be explored.

For instance, consider the work of a "computer user support specialist", a job we will use as a running example in this paper. It may soon be (if it is not already) feasible to develop an automated system to answer computer users' support questions [6]. However, to be functional, such a system needs to fit the complex work of an organization. Someone must identify that there is a problem, collect relevant information to input to the system, explain the diagnosis to the user, implement the fix, and so on. All of this surrounding work needs to adapt to an automated computer support system (and vice versa).

We argue that ML-based systems are capable of handling a greater range of decision-making tasks. As a result, there may be many new possible relationships between workers and automated systems. The research question we address in this paper is: "what are the different patterns of relationship between human and MLbased automated systems?" We answer this question by developing a simple conceptual model of task automation and using it to analyze the relationships between humans and machines in bellwether settings drawn from published reports. Knowing the answer to this question is important to expand our conversations about AI beyond an all-or-nothing focus on automation and work and to encourage thinking about alternative ways to deploy systems and improve work design.

\section{Theory}

In this section, we develop a conceptual model of task automation to analyze how ML-based systems might have an impact on the human jobs. We first present a model for analyzing jobs, then discuss novel features of ML, then combine these perspectives to develop a set of issues to consider while analyzing the relationship between work design and technology.

\subsection{Work design}

We start by presenting our perspective on human work. In their jobs, most workers do a variety of dif- 
ferent actions that might be more or less susceptible to automation. As noted above, a job is therefore not the right level at which to understand the impacts of technology. We follow the job analysis approach [7] in considering a job "an aggregation of tasks assigned to a worker" [8]. In turn, a "task represents certain processes in which the worker, through his or her actions, transforms inputs into outputs meaningful to the goals of the job by using tools, equipment, or work aids" [8]. The Employment and Training Administration of the U.S. Department of Labor has a database called O*Net that provides detailed information about jobs, including the comprised tasks. For example, the top three tasks (of 16) given for a "computer user support specialist"1 are:

1. Answer user inquiries regarding computer software or hardware operation to resolve problems.

2. Oversee the daily performance of computer systems.

3. Read technical manuals, confer with users, or conduct computer diagnostics to investigate and resolve problems or to provide technical assistance and support.

In summary, the design of work is defined as "the content and organization of one's work tasks, activities, relationship and responsibilities" [9].

\subsection{Automating tasks}

We next consider how a new technology might enable a task to be executed by a worker in a different way or to be completely automated. By automation, we mean the capability of a system to perform some task without human involvement. Since we are focusing in this paper on the applications of ML, we restrict our analysis to information-processing tasks, i.e., we do not consider the impact of robots on physical work.

For this analysis, we apply a model from [10] that suggests decomposing tasks into a "simple four-stage view of human information processing": 1) information acquisition; 2) information analysis; 3) decision and action selection; and 4) action implementation. Essentially, the steps of information analysis and decision and action selection match a particular action to a given set of input conditions. For each step, we consider if it can be partly or fully automated, meaning that the particular step can be done by a system without human intervention. Given this model of a task, we characterize tasks along the dimensions of inputs, outputs and the mapping between them.

\footnotetext{
${ }^{1}$ https://www. onetonline.org/link/details / $15-1151.00$
}

2.2.1. Inputs Inputs are the information acquired for the task. Drawing on the definition proposed for big data, we characterize these inputs by the volume, velocity and variety of the information acquired. For example, some tasks like answering user computer support queries might have a high volume of requests in total, arriving at a high rate during certain times of the day (velocity) with a high variety of different queries, some more common, but with lots of exceptions.

2.2.2. Outputs Outputs can also be characterized by the $3 \mathrm{Vs}$. In this case, by variety we consider a number of possible actions to be selected among. The decision could be binary (e.g., parole/no parole for a judicial decision or cancer/no cancer for a radiological screening) or of very high dimensionality (e.g., hundreds of possible replies in a customer-support setting or for a more complicated medical diagnosis). Again, we also need to consider the distribution of the outputs, whether some outputs are more common than others, i.e., the proportion of exceptions [11].

2.2.3. Mapping between inputs and outputs Finally, we consider the complexity of the decision rules that connect inputs and outputs, which covers the steps of information analysis and decision and action selection. These rules could be very regular (i.e., high analyzability [11]) or very irregular (low analyzability).

2.2.4. Dynamics of tasks The above discussion has considered inputs, outputs and the mapping as static, but there could also be a dynamic aspect. For example, the nature of the inputs and outputs could change over time rather than being static and pre-given. Tasks are most likely repeated, so the information acquired as inputs could include feedback from prior rounds. And the mapping rules could evolve as system learns or as inputs and outputs change.

2.2.5. Tasks and automation Given this model, we can consider what parts of the task-framework are automated. We have suggested that information acquisition has to be at least partly automated for the task to be automatable, but performance of task might also rely on information held by humans. Similarly, information analysis and decision selection could be done by humans, automation or some mix, as is the case for action implementation. These combinations yield a number of patterns of automation. Pacaux et al. [12] identified 10 
levels, some with sublevels. Level 1 is no automation, i.e., all four steps performed by a human, while level 10 is total automation, i.e., all four steps performed by a machine without human intervention. As we assume that information acquisition and decision implementation are at least partly automated, we consider a subset of the 10 levels, which will be presented below.

\subsection{Technical drivers for automation}

Given this framework, automation enhances its characteristics, and indeed likely has already happened, for tasks with a high volume of data, arriving with high velocity and where there is a high need for replicability of the decision making. For example, credit card fraud detection has to be automated because of the volume of transactions and the need to make the decision quickly and accurately. Similarly, stock trading is largely automated. We recognize two technological drivers that support an increase in automation.

2.3.1. Digitization The first driver is digitization: increasingly more data and interactions are digital. The greater penetration of digitized data implies that the data acquisition and decision implementation steps of our task model are done via a system, increasing the range of tasks executable through machine processing. For example, consider our computer user support specialist answering user inquires. These inquires could happen in face-to-face or over the phone. However, if they are submitted via a computer system (digitally), then they can be processed by the system, opening the potential for automation. Similarly, if the users' computer systems are networked an automated system could act on them directly to address problems, expanding automation to include decision implementation.

2.3.2. Machine learning The second issue is about what can be automated in the intervening steps of information analysis and solution/action selection. Situations with very irregular rules - i.e., low analyzability [11] resist automation due to the variety of data and exceptions to the connection between input and output.

What recently changed is that the capabilities of automated systems has improved. In the past, decision making was automated with a set of rules: if some parameter or combination of parameters have particular values, then a particular decision is taken. ML system provide new capabilities for complex pattern recognition. Rather than having to make explicit "if-then" rules, a system can learn the appropriate outputs given a large set of training examples (input-output pairs). For ex- ample, given a sufficient volume of known fraudulent and legitimate transactions, a system could learn which transaction's characteristics suggest a fraudulent activity. Furthermore, a system can learn from cases over time and so continue to improve. Having been trained, an ML-system given some entity will give a score for how well it matches the pattern. The score can be converted to a decision by comparing it to a threshold, fixed or compared to others in a pool. Applying ML, a system can learn to identify solutions that were not coded ex-ante by humans and thus handle less analyzable mappings between inputs and outputs. Even where tasks are already automated, automation can be improved by refining the quality of the mapping from inputs to outputs.

However, ML systems have distinctive characteristics that are unlike prior systems for supporting or automating work. A first major difference is that ML performance depends heavily on the quantity and quality of data available for the training. ML requires a large training dataset, with high volume of inputs and outputs. Furthermore, as systems are reliant on data, they often exhibit hybrid agency, combining human and machine actions; human to generate an initial dataset and then further ML-based actions, meaning that initial human biases may be amplified. Second, the results of ML are most often probabilistic: e.g., when classifying an unknown case, an ML system likely provides probabilities that the unknown case fits one of the known categories rather than a definitive answer. Finally, many ML systems are opaque: unable to explain why a particular output was selected. Overall, ML systems behave quite differently than programmed systems.

2.3.3. When is automation feasible? We suggested above that the trends towards digitization and the increased capabilities of ML-systems point towards increased possibilities for automation. However, the possibilities for automation depends on nature of the task, particularly the proportion of exceptions in the inputs and outputs and the stability and analyzability of the mapping between them [11]. Stable, routine tasks, those with high analyzability and few exceptions, have little or no need for information analysis or decision and action selection, meaning that the worker can just implement the actions. Such tasks are also very automatable.

If the task has low analyzability, but few exceptions, then analysis is hard, but the selection of actions is from limited range. These tasks may be increasing amenable to automation with the capability of ML-systems to learn patterns. For tasks with high analyzability but many exceptions, analysis may be easy, suggesting automation, but large number of choices for action may be 
problematic for ML, both in ensuring that the training data are complete and for achieving the necessary precision. Non-routine tasks are both low in analyzability and high in exceptions, suggesting that automation will be difficult. And finally, unstable tasks, ones for which the inputs, outputs or the mapping evolve over time, will also be challenging to automate.

\subsection{Task interdependencies}

The analysis above has considered an individual task. But jobs are typically collections of tasks, not just one, and furthermore, people doing a job typically have to interact with others. As a result, the impact of using ML for a task will propagate beyond the boundaries of the task itself.

To analyze multiple tasks, we consider how a particular task is interdependent with others, defined as "the extent to which the inputs, processes, or outputs of the tasks affect or depend on the inputs, processes, or outputs of other tasks within the same job" [8]. For example, the second task in the list for a computer user support specialist is to monitor system performance. It may be that handling problem reports from users is helpful to see when a system has changed, because the kinds of problems change. If that first task is entirely automated, the specialists will need to develop new ways to get information about the systems.

We can also consider interdependencies between tasks that compose different jobs. An isolated task might be automated with few consequences, while one that interacts with many other jobs will be more problematic. While this perspective is quite common in studies of organizational design, it is interesting to note that the $\mathrm{O} *$ Net database does not record task interdependencies or what other jobs a job interacts with.

To analyze interdependencies, we adopt a coordination theory approach [13, 14]. Malone and Crowston [14] analyzed group action in terms of actors performing interdependent tasks to achieve some goal [13]. These tasks might require or create various resources. The actors face coordination problems arising from dependencies that constrain how tasks can be performed.

The key point in coordination theory is that the dependencies create problems (or possible synergies) that may require additional work to manage, what Malone and Crowston [14] called coordination mechanisms. Studying coordination thus means analyzing the dependencies that emerge among the tasks in a system and identifying how those dependencies are managed. As the pattern of dependencies among tasks changes, we expect to see corresponding shifts in the needed coordination mechanisms.
Dependencies between a task and a resource arise because a task uses or creates a resource. In particular, the steps in the tasks need to be carried out by some actor, either a human or a machine, creating a need for a task assignment mechanism to identify which actor should work on which task. For example, if expertise is necessary to perform a given task (i.e., there is a task-actor dependency), then an actor with that expertise must be identified and the task assigned to him or her. In our analyses, we can consider whether the human assigns work to a system or vice versa.

Use of common resources among tasks leads to dependencies between the tasks that use or create the resource. These dependencies come in three kinds.

First, producer-consumer or flow dependencies match Thompson's sequential dependency [15]: one task creates a resource that a second uses. Flow dependencies imply the need to manage the usability of the resource and the timing and location of its availability (i.e., a flow dependency has three aspects). Considering usability for example, we might consider whether the machine adapts to the needs of the human or vice versa.

Second, a shared-output or fit dependence occurs when two activities collaborate in the creation of an output (in the case where the output is identical, there is potential synergy, since the duplicate work can be avoided). Again, the integration of different outputs might be done by a human or a machine.

Finally, a shared-input dependency emerges among activities that use of a common resource (like Thompson's pooled dependency [15]). One version of this problem is a task needing an actor to to work on it. Note that information as a resource is easily shareable, which can ease management of shared-input dependencies, but simultaneously creates a different dependency of ensuring that different tasks are working with the same version of the data.

\subsection{Impact of automation}

Decisions made about work design will have implications for the nature of the jobs that are created. Studies have identified a wide set of factors that characterize work designs (e.g., level of autonomy, feedback, interdependence, skill variety, physical), diverse outcomes of work design for workers (e.g., attitudinal outcomes such as satisfaction or motivation, behavioral outcomes such as performance or turnover, cognitive outcomes such as learning or identify and well being outcomes, such as anxiety, stress or burnout) and finally, mechanisms that link work design and work outcomes. Taken together, the outcomes for the workers have implications for organizations (e.g., overall productivity, skill and training 
needs, costs). More recent work has expanded work design to consider the impact of group work design and group outcomes as well, e.g., [16].

A particular concern with automation is the ability of those interacting with the automated systems to understand what the systems are doing and to intervene if needed. For instance, there have been many studies of pilots interacting with intelligent autopilot systems that largely automate the job of flying. One outcome of this work is the identification of the problem of automation surprises [17], when the human operator loses track of the state of the automated system and so is surprised by unexpected or inappropriate actions or has difficulty taking over in a crisis. Automation surprises seem particular problematic for ML-systems, as the precise reason for an answer often can not be pinpointed.

\section{Methods}

\subsection{Data}

Our goal in this paper is to use our conceptual model to analyze changes that have already taken place in bellwether settings. In other words, we adopt William Gibson's perspective that, "The future is already here-it's just not evenly distributed". To gather data about settings in which the future is here, we searched for published articles in the popular press about jobs affected by automated systems.

To gather a broad sample, we searched for articles in English, Italian and Russian, searching Google Scholar for recent articles that mentioned the keywords "AI" or "artificial intelligence" or "machine learning" and "example cases" or "work design" or "employment" or "future of work". The results were manually filtered to identify popular press articles that were relevant to the topic. We developed a collection of 94 articles in English, 21 in Italian and 16 in Russian.

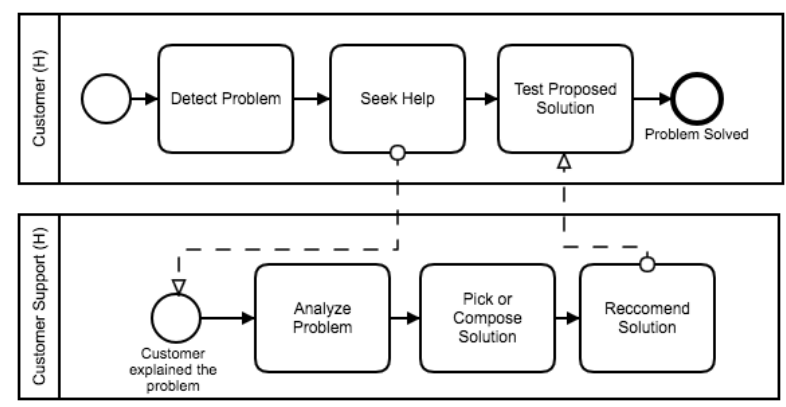

Figure 1. Level 1 automation: No technology support

\subsection{Analysis}

The work presented in this article represents a pilot analysis of the data. We examined the articles to identify the nature of the tasks impacted by ML along the dimension of our conceptual model. Doing so allowed us to sort examples into a number of patterns. For each pattern, we use Business Process Modelling Notation (BPMN) to represent jobs and compare them (e.g., how was a task performed when fully human-driven and how is the same task designed when ML is introduced).

\section{Findings about task automation}

In this section, we present 5 patterns for the performance of tasks, 3 including automation, based on the framework presented above.

\subsection{No automation}

We start with two base cases: an entirely nonautomated system (Pacuax et al.'s [12] level 1) and a task with some technology support but not for analysis or decision and action selection (Pacuax et al.'s [12] level (2)). Level 1 means a human performing user computer support in person, as shown in Figure 1. All the steps of the task are done by the human. Also shown is how this task relates to another task, in this case, the computer user's interrupted task. This pattern could represent any kind of information processing task done manually.

A more likely scenario for user computer support involves some technology, even if there is no automation. For example, receiving and responding to problem reports via email adds digitization but not automation. In our framework, this change is represented by having just the first and last steps of information acquisition and action implementation supported by a system, as shown in Figure 2. In this case, the automated system provides information to support the human doing the task. Specifically, the person can collect information with system support (e.g., email or a problem reporting system) and take action through the system (e.g., send an email in response). We might also imagine support for other steps, e.g., a searchable database of problems to use in responding to a problem, but again, no automated steps.

We acknowledge that this model is quite simplified. For example, even for computer system support, it is unlikely that the information needed will simply be provided. Instead, one of the skills of a computer support specialist is to be able to interview the user to determine the relevant details. Nevertheless, the model allows us to distinguish several patterns of automation. 


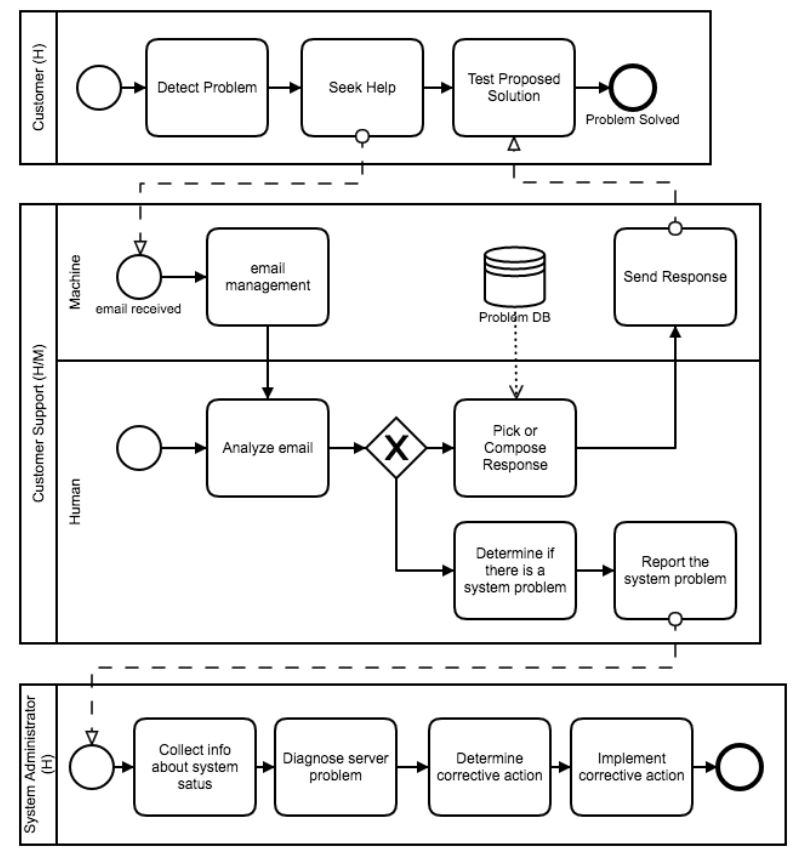

Figure 2. Level 2 automation: Digitization but not automation

\subsection{Decision support}

The first step in automation is that information analysis and action selection is supported for a subset of the cases encountered. We also assume the previous level of automation, that is that the information acquisition is digitized, so system has access to the data. In this pattern, the system provides decision support (Pacuax et al.'s [12] level 3-4), meaning that the system can analyze the data and suggest one (level 4) or a few (level 3) responses, while leaving it up to the user to chose whether to accept and implement that response. This model is shown in Figure 3.

For example, in 2017 Google introduced a function called "Smart Reply", where the system suggests three possible answers to a message, based on its analysis of a large, anonymized body of other emails. The user can choose to pick the suggested answer, to customize it or simply to ignore the system's recommendations [18].

A system can support sales by providing useful information to finalize a potential sale, but leaving it to the salesperson to decide if and how to use those suggestions. Udacity, an education company providing online courses, built a digital sales system to assist humans. When a potential customer asks a question, the system suggests an appropriate response that a salesperson can follow or customize. With this digital sale assistant, it is reported that the sales team was able to manage twice as many potential customers and to convert to actual cus-

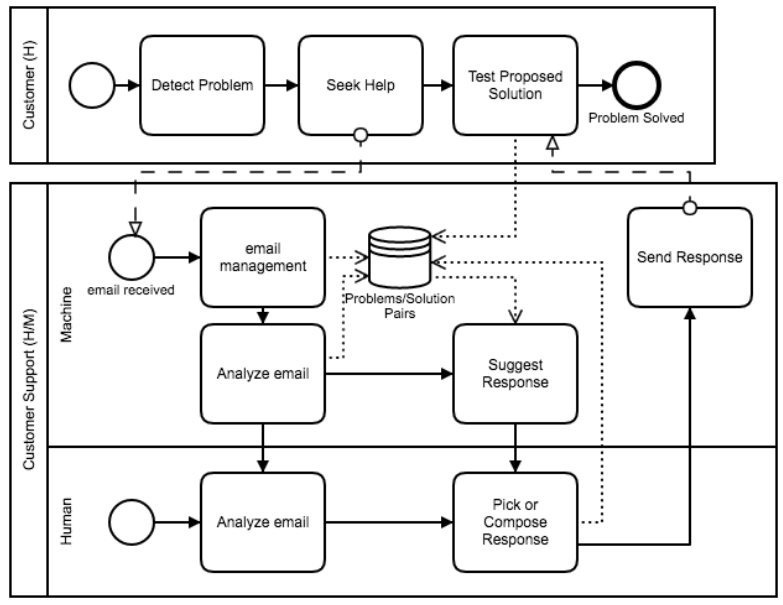

Figure 3. Level 3-4 automation: Decision support

tomers a higher percentage of inquiries [19].

NDA-Lynn is a system that can evaluate a nondisclosure agreement, identifying clauses that are too strict, unclear or not coherent with the whole agreement. Such a system could drastically reduce the time needed to read the document, allowing the lawyers to focus on reviewing the clauses that have been flagged as problematic by the system [20].

In the US judicial system, some courts rely on ML systems to determine the probability that a defendant will commit another crime or will appear for his/her court date. Kleinberg et al. [21] suggest AI systems are able to improve trial accuracy by warning judges when a decision they are about to take (e.g., allowing or not allowing parole) is likely to be wrong.

Systems are also increasingly used by recruiting agencies to match companies and job-seekers. Woo [22] developed a system to simplify the employment process, matching passive job-seekers' profile and skills to employers' needs. The system signals potential matches to the individual, who can choose whether or not to pursue the selected opportunity.

Many decision support systems have been designed for medical diagnosis. For example, a radiology system could provide warnings of possible anomalies in images to help separate urgent cases from those that can wait, while still requiring a human doctor's decision [23].

The tasks in this category share some common features. A prerequisite is a high volume of input data with matched outputs to make feasible assembling a training set. Note that in some of these cases, the decision implementation step is not automated, meaning that the human both decides and carries out the decision. The locus of control stays with the human through out the task and the human remains mostly embedded in the 
task rather than peripheral. The implementation of the system as decision support suggests that the human decision maker may have information that's not available to the machine.

We expect to see this pattern in cases where acquisition of information and decision implementation are mostly digitized, and where the input has high volume and velocity, so automation makes sense and is feasible, but the variety is high, so that pursuing complete automation is not feasible. Further, if the level of exceptions is high, identifying the relation between inputs and outputs is difficult, so human input is still needed.

Even though the system in the end defers to the human, implementation may raise some concerns. First is auditability: how does human know the system made the right recommendation? Will the human worker be able to recognize when to ignore the system? Second is deskilling: i.e., does relying on the system reduce the skill of the human in the long run?

\subsection{Blended decision making}

The next step in automation presumes that for some set of the cases the machine can take and implement decisions by itself, what Pacuax et al.'s [12] called level 6, blended decision making. This model is shown in Figure 4. For example, for user computer support, the system would automatically answer requests for which it was confident of an answer (e.g., the easy cases), deferring others to the human.

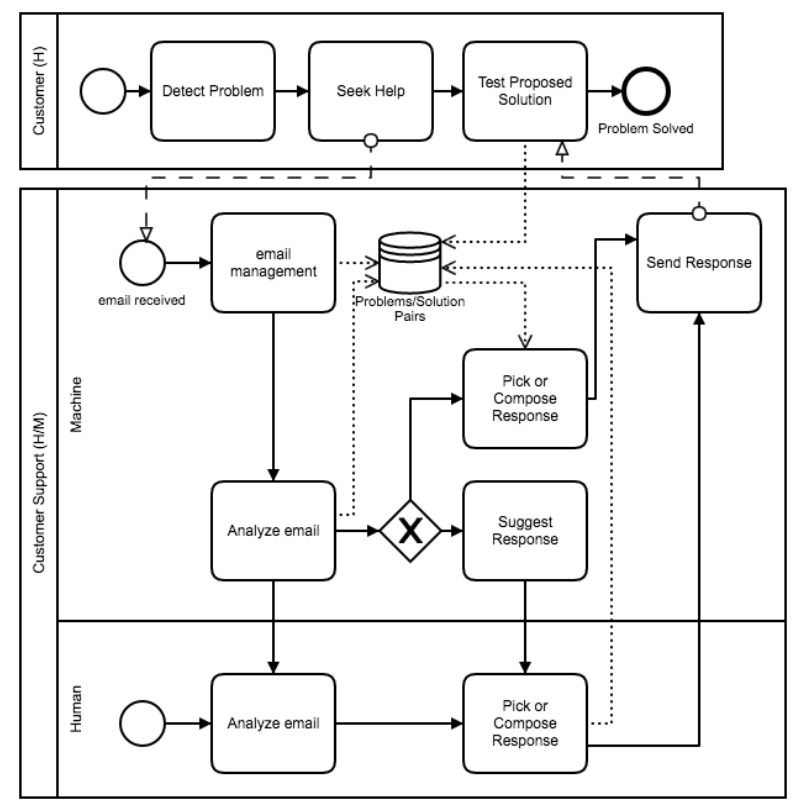

Figure 4. Level 6 automation: Blended decision making
Examining the process models, we note that we are adding a new step, training the system to assign some tasks to a human and some to the machine. To do so, the system has to know what it can do, as well as doing it. An alternative approach for cases with low individual volume is to have the human to decide which cases the system should do, as shown in Figure 5. For example, an architect might decide which aspects of a building design are sufficiently routine to entrust to an automated design system, and which should be done by humans.

An example from the judicial system is the plan proposed by the Moscow City Court to use a system to prepare judicial decisions on so-called "indisputable cases". Human judges would intervene in such trials only in case of complaints about system-supported decisions, enabling them to focus on more controversial cases [24].

For this pattern, again a large volume of training data will be needed. Furthermore, the system need to be trained to recognize exceptions that should be deferred to the human. An interesting possibility in this case is that the ML algorithms can be periodically retrained on the cases performed by the human. In this way, the performance of the system can be improved, perhaps allowing it to take on more tasks.

The issues noted above for level 3-4 take on more urgency in this pattern because some actions will be implemented automatically. For example, how will human workers know what the system is doing and how will they be able to intervene if something goes wrong? Relying on customers to complain seems problematic.

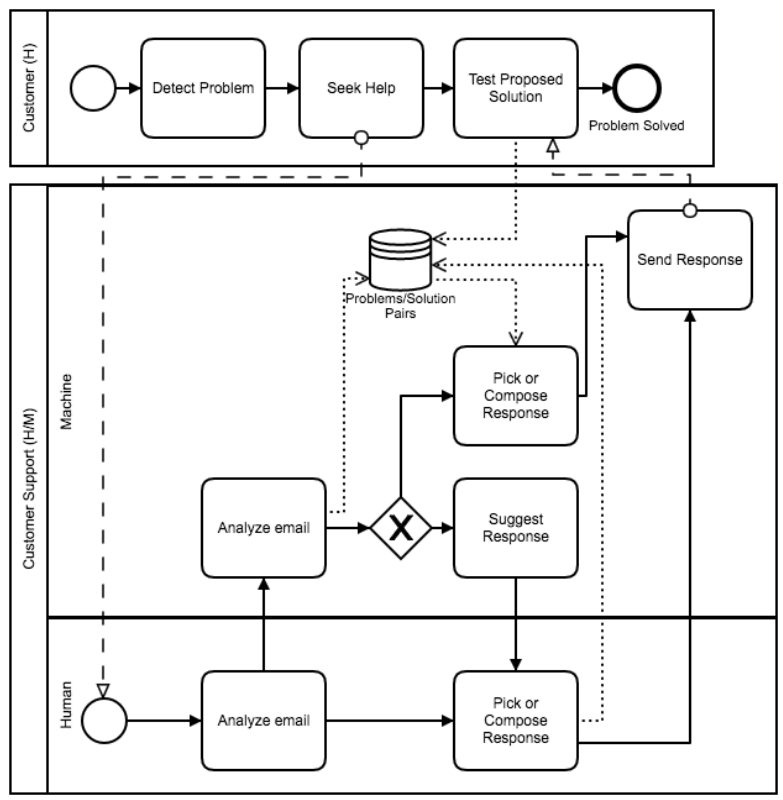

Figure 5. Level 6 automation: Blended decision making with human allocation of effort 
The question of deskilling takes on a different perspective in this pattern. It seems likely that the tasks the machine will do are the more straightforward one. A resulting concern is if those tasks constitute the entry-level job for the profession, e.g., the tasks that an architect delegates to the machine are those that would have been done by a junior architect. If these are instead done by the system, how does someone learn to do the task and enter the profession?

\subsection{Task automation}

Finally, we consider the case of complete automation (Pacuax et al.'s [12] level 10; their levels 7-9 are where the system seeks human input before going ahead with a decision). In this case, the system performs all of the steps above for all cases. If the implementation of the decision is automated, then the whole task can be done automatically, as shown in Figure 6. For example, computer user support might be provided by an automated system that parses a user's emailed report and automatically replies. In this pattern, the automated system does the task, while the role of the human is to monitor and possibly tune parameters to improve performance.

This pattern describes many already automated systems. In the stock market, fully automated trading systems are constantly buying and selling stocks on the base of their own predictive models and criteria. In Japan, Mizuho Financial Group recently introduced an algorithm-based trading service to predict how prices will change over an hour and find the best time to trade [25]. Credit card companies broadly rely on anti-fraud systems for increasing the accuracy of automated and real-time approvals while reducing false declines [26].

We expect to see this pattern when the analyzability of the task is high and there are few exceptions (i.e., when the task is more routine), because the system has to be to handle all of the cases. As ML is applied, we expect systems to be able to handle tasks with lower analyzability, but not a high numbers of exceptions.

A question in designing such systems is the locus of control. Even if decision implementation is not automated and so is carried out by a person, a system could follow this pattern if the human is not given the authority to override the system by implementing a different decision. Alternately, even if the system is entirely automated, there may also be ways that a human can intervene in a particular decision for a specific case. For example, in social media users' reports of inappropriate behavior (or "bad" advertisements) are automatically checked by the system and appropriate measure are taken, however in specific cases, an editor can intervene to re-calibrate the automated answer.

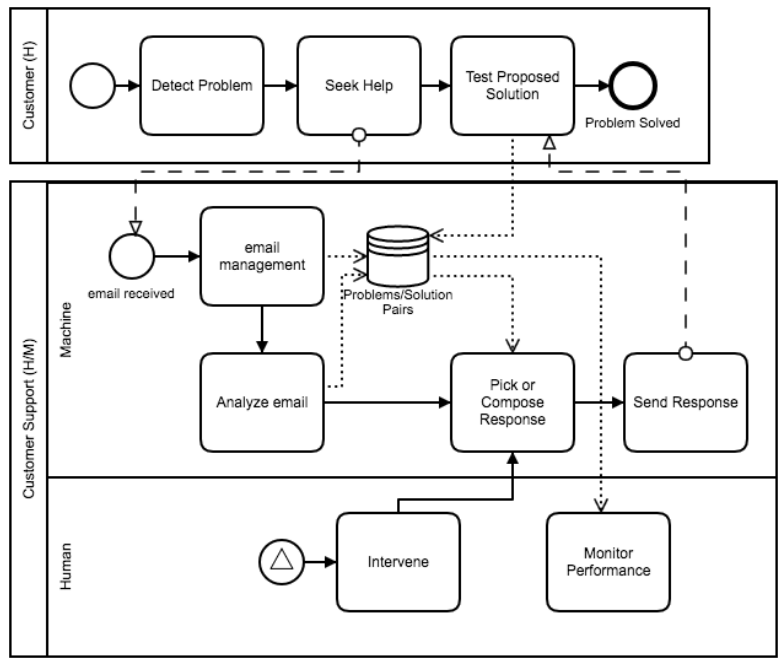

Figure 6. Level 10 automation: Complete automation with possible interventions or oversight

As more tasks are automated, the role of humans in a system of tasks may reduce. In a study of bank automation, Adler [27] described the process of peripheralization, in which automated processes relegate humans to the periphery of the processing: entering data into the system or monitoring its performance. Interestingly, peripheralization can have paradoxical effects. While automating a process might be expected to deskill workers who no longer need to know how to perform the tasks in the process, Adler [27] observed that it can also increase skill demands, as workers need to be able to comprehend the entire automation process to understand how their input affect the system and to debug problems.

As a result, this pattern poses the greatest risk for automation surprises. The model envisions a new role for humans as system monitors and meta-designers (i.e., designing the tasks for the machine to perform and setting the parameters), but how does human who's monitoring the system understand what it's doing and the impact of the parameters they can tweak?

\subsection{Jobs with multiple tasks}

We now switch our consideration to the impact of ML-based systems across multiple tasks, within or across jobs. We consider that each task uses some information as an input and creates some information as an output. For example, our computer system support specialist takes (or elicits) problem reports as input and produces recommended solutions as an output. The input and outputs are provided from some other task, as shown in the various figures above.

To illustrate, we consider issues specifically with 
flow dependencies. A flow dependency refers to the case where the output of one task is the input to the next. As discussed above, flow dependencies imply three subdependencies: ensuring that the resource created is what is needed, that it is available when it is needed and that the resource is transferred to where it is needed.

Consider the impact of automating one of the two tasks. An example is an automated legal discovery system tasked with finding documents in a very large collection to be used by lawyer in assembling a pending legal case. The automation is of the upstream task, so a question is how automated system knows what output is needed by downstream tasks. Coordination theory suggests approach such as standardization of the system asking the users what information they want. Another approach is for the users to give feedback on the documents they're provided to refine the search.

Contrariwise, the downstream system might be the one that is automated. For example, the task of taking an $\mathrm{X}$-ray feeds a task of interpreting the X-ray. If the later task is automated, then it seems likely that the X-ray images provided would have to be more standardized to meet the needs of the system. The need for standardized inputs may be problematic if the information is hard to express. For example, the task of finding a house (part of the job of a real estate agents) seems straightforward but in fact can involve many emotional criteria that can be hard for buyers to articulate. Part of the skill of an agent is eliciting these criteria. We noted above similarly that extracting useful problem reports is an important skill for a computer user support specialist. However, it is more challenging to develop an automated system to elicit information from a user than to parse emails.

This analysis can be extended to the next of tasks. For example, the X-ray diagnosis task has a downstream task, e.g., sending the patient for additional care. How the information about the diagnosis should be presented will depend on the needs of that task.

Finally, we note that the flows may be implicit rather than explicit. For example, as noted above, handling customer problem reports may be a way to learn about the status of systems, e.g., identifying problems by when the problem reports change. As a result, automating problem resolution may remove an important source of information. The opposite is also possible: if oversight is automated, the specialists may be unaware of ongoing system problems that affect users.

\section{Discussion}

The patterns of automation discussed above differ in the level of automation, from individual support to process automation. Much of the rhetoric has focused on the latter case, but research is needed also on how to effectively do the former. As well, many tasks are performed by or with teams rather than solely by individuals, so research is needed to identify how a system can be an effective team member.

Despite the differences, the patterns have a number of commonalities. First is a need for a sufficient volume and quality of training materials and a sufficient regularity of the relationship between inputs and outputs. If the task has many exceptions, an ML might not be able to learn them, suggesting a decision support or blended decision making pattern. A particular challenge to the blended decision making pattern is the ability of the ML to know when it does not know and should defer the case to the human. Finally, if the task is not stable, automation will be challenging.

A second common issue has been transparency of decision making. In all of the models, there is a need for human workers to maintain awareness of the system's performance. A third issue as automation increases is to identify the circumstances under which is is reasonable for the humans to be able to veto the machine. These interventions may decrease the reproducibility of the task (and so face managerial opposition), but they also acknowledge that the automated system may not have complete information. These two concerns (visibility and agency) are tightly coupled, because the former is necessary to be able to implement the latter.

We note that a human worker may technically have the authority to make the final decision (i.e., the system nominally follows the decision support pattern) but face obstacles to exercising the authority, resulting in practice in complete automation. The pressure to follow the system could be from internal management or external forces. For example, a doctor who decides to ignore the advice of a medical expert system could risk a suit for malpractice for not following the encapsulated "best practice". In such a situation, a doctor might feel forced to cede authority to the system.

\section{Conclusion}

The analysis of the articles in this paper was a pilot application of the conceptual framework. Future work includes a more systematic analysis of the articles. A shortcoming of relying on popular press articles is that few discuss the impacts of using the system on workers in any detail. More detailed case studies will likely be needed to establish these connections. Finally, further analysis is needed of multiple intersecting tasks, e.g., when tasks are composed of multiple subtasks.

The main message of this paper is that contrary to a rhetoric of the march of automation, there are a variety 
of options for how automated systems can be used with differing impacts on jobs. The decision about which pattern to follow is partly driven by nature of tasks and system capabilities. But designers should resist technological determinism and be aware of the impacts of managerial decisions about how technologies should be deployed, especially about the desired locus of decision making. Designers should strive for a fit between system characteristics and the characteristics of the setting in which the automation is introduced.

\section{References}

[1] P. K. Chan and S. J. Stolfo, "Toward scalable learning with non-uniform class and cost distributions: A case study in credit card fraud detection.," in $K D D$, vol. 98, pp. 164-168, 1998.

[2] A. Esteva, B. Kuprel, R. A. Novoa, J. Ko, S. M. Swetter, H. M. Blau, and S. Thrun, "Dermatologist-level classification of skin cancer with deep neural networks," Nature, vol. 542, no. 7639, p. 115, 2017.

[3] R. Berk and J. Hyatt, "Machine learning forecasts of risk to inform sentencing decisions," Federal Sentencing Reporter, vol. 27, no. 4, pp. 222-228, 2015.

[4] M. Chui, J. Manyika, and M. Miremadi, "Four fundamentals of workplace automation," McKinsey Quarterly, vol. 29, no. 3, pp. 1-9, 2015.

[5] World Economic Forum, The future of jobs: Employment, skills and workforce strategy for the fourth industrial revolution. 2016.

[6] R. P. Masongsong and M. A. E. Damian, "Help desk management system," in Proceedings of the World Congress on Engineering and Computer Science, vol. 1, 2016.

[7] United States. Employment and Training Administration and U. S. Department of Labor, The Revised Handbook for Analyzing Jobs. Career Reference Books, JIST Works Incorporated, 1991.

[8] C.-S. Wong and M. A. Campion, "Development and test of a task level model of motivational job design.," Journal of Applied Psychology, vol. 76, no. 6, p. 825, 1991.

[9] S. K. Parker, "Beyond motivation: Job and work design for development, health, ambidexterity, and more," Annual Review of Psychology, vol. 65, pp. 661-691, 2014.

[10] R. Parasuraman, T. B. Sheridan, and C. D. Wickens, "A model for types and levels of human interaction with automation," IEEE Transactions on Systems, Man, And Cybernetics-Part A: Systems and Humans, vol. 30, no. 3, pp. 286-297, 2000.

[11] C. Perrow, "A framework for the comparative analysis of organizations," American Sociological Review, vol. 32, pp. 194-208, 1967.

[12] M.-P. Pacaux, S. Debernard, A. Godin, B. Rajaonah, F. Anceaux, and F. Vanderhaegen, "Levels of automation and human-machine cooperation: Application to human-robot interaction," in Proceedings of the 18th World Congress of The International Federation of Automatic Control, vol. 44, pp. 6484-6492, 2011.

[13] K. Crowston and C. S. Osborn, "A coordination theory approach to process description and redesign," in $O r$ ganizing Business Knowledge: The MIT Process Handbook (T. W. Malone, K. Crowston, and G. Herman, eds.), Cambridge, MA: MIT Press, 2003.
[14] T. W. Malone and K. Crowston, "The interdisciplinary study of coordination," Computing Surveys, vol. 26, no. 1, pp. 87-119, 1994.

[15] J. D. Thompson, Organizations in Action: Social Science Bases of Administrative Theory. New York: McGrawHill, 1967.

[16] F. P. Morgeson and S. E. Humphrey, "Job and team design: Toward a more integrative conceptualization of work design," in Research in Personnel and Human Resources Management, pp. 39-91, 2008.

[17] N. B. Sarter, D. D. Woods, and C. E. Billings, "Automation surprises," Handbook of Human Factors and Ergonomics, vol. 2, pp. 1926-1943, 1997.

[18] A. Kannan, K. Kurach, S. Ravi, T. Kaufman, B. Miklos, G. Corrado, A. Tomkins, L. Lukacs, M. Ganea, P. Young, and V. Ramavajjala, "Smart reply: Automated response suggestion for email," in Proceedings of the ACM SIGKDD Conference on Knowledge Discovery and Data Mining (KDD) (2016)., 2016.

[19] E. Anthes, "The shape of work to come," Nature News, vol. 550, no. 7676, p. 316, 2017.

[20] A. Tauber, "This AI checks NDAs for free: And offers a grim glimpse of the future." https://thenextweb.com/artificial-intelligence/2018/02 /27/ai-checks-ndas-free-offers-grim-glimpse-future/.

[21] J. Kleinberg, H. Lakkaraju, J. Leskovec, J. Ludwig, and S. Mullainathan, "Human decisions and machine predictions," The Quarterly Journal of Economics, vol. 133, no. 1, pp. 237-293, 2017.

[22] M. Munford, "Woo launches the first AI-powered headhunter for employers-She's called Helena." https://www.forbes.com/sites/montymunford/2017/11/27 /woo-launches-the-first-ai-powered-headhunter-foremployers-shes-called-helena/\#409adb9a67f8.

[23] D. Pearson, "Artificial intelligence in radiology: The game-changer on everyone's mind." https://www.radiologybusiness.com/topics/technologymanagement/artificial-intelligence-radiology-gamechanger-everyones-mind.

[24] G. Sysoev, "Artificial intelligence will help Moscow judges make decisions." https://lenta.ru/news/2018/05 /29/judge/.

[25] T. Hyuga, "Mizuho is said to offer AI trading before MiFID overhaul." https://www.bloomberg.com /news/articles/2017-09-20/mizuho-is-said-to-offer-aitrading-service-before-mifid-overhaul.

[26] M. Cochrane, "How MasterCard is using AI to improve the accuracy of its fraud protection." http://www.businessinsider.com/mastercard-artificialintelligence-fraud-protection-2017-1 ?IR=T.

[27] P. Adler, "New technologies, new skills," California Management Review, vol. 29, no. 1, pp. 9-28, 1986. 